\title{
Exosome and Melatonin Additively Attenuates Inflammation by Transferring miR-34a, miR-124, and miR-135b
}

\author{
June Seok Heo $\mathbb{D}^{1},{ }^{1}$ Ja-Yun Lim, ${ }^{2}$ Dae Wui Yoon $\mathbb{D}^{3},{ }^{3}$ Sangshin Pyo, ${ }^{4}$ and Jinkwan Kim $\mathbb{D}^{3}$ \\ ${ }^{1}$ Cell Therapy Center, Severance Hospital, Seoul 03722, Republic of Korea \\ ${ }^{2}$ Department of Integrated Biomedical and Life Sciences, Graduate School, Korea University, Seoul 02841, Republic of Korea \\ ${ }^{3}$ Department of Biomedical Laboratory Science, College of Health Science, Jungwon University, Goesan 28024, Republic of Korea \\ ${ }^{4}$ Department of Clinical Laboratory Science, Daejeon Institute of Science and Technology, Daejeon 35408, Republic of Korea
}

Correspondence should be addressed to Jinkwan Kim; jkkim@jwu.ac.kr

Received 7 August 2020; Accepted 16 November 2020; Published 24 November 2020

Academic Editor: Bruna Sinjari

Copyright (c) 2020 June Seok Heo et al. This is an open access article distributed under the Creative Commons Attribution License, which permits unrestricted use, distribution, and reproduction in any medium, provided the original work is properly cited.

\begin{abstract}
The positive effects of mesenchymal stem cells (MSCs) are primarily activated through molecular secretions known as paracrine activity, which regulates the function of various cell types including immune cells. Accumulating evidence shows that exosomes of soluble factors released from MSCs are potential alternative agents for stem cell-based therapy, although the exact underlying mechanism has not been elucidated. The purpose of this study was to evaluate the potential effects of exosomes produced by adipose-derived MSCs and to examine the changes in anti-inflammatory genes in concurrence with the polarization of M2 macrophages in cellular models ex vivo. Isolated exosomes were used to investigate the inflammatory modulation in proinflammatory cytokine-treated fibroblasts and THP-1 cells. The anti-inflammatory mRNA expression associated with M2 macrophages was significantly upregulated after exosome treatment in an interferon gamma and tumor necrosis factor alphatreated inflammatory environment. Furthermore, melatonin-stimulated exosomes exerted superior anti-inflammatory modulation via exosomal miRNAs miR-34a, miR-124, and miR-135b, compared with exosomes. Our results indicate that melatonin-stimulated exosomes originating from adipose-derived MSCs are safe and efficient tools for regenerative medicine to treat inflammatory diseases.
\end{abstract}

\section{Introduction}

Mesenchymal stem cells (MSCs) are promising cell sources owing to their multipotent and immunosuppressive properties [1]. Accumulating evidence shows that MSCs exert antiinflammatory effects by modulating immune cells and the inflammatory environment [2]. Hence, clinical applications using various types of MSCs have been actively conducted to develop new treatments, especially for inflammation-related disorders such as graft-versus-host disease, multiple sclerosis, joint diseases, inflammatory bowel diseases, inflammatory airway disease, and pulmonary diseases [3]. Although the effectiveness of MSCs is predominantly associated with paracrine effects rather than direct differentiation or engraftment, the underlying mechanisms of MSC therapy are unclear [4].

Recently, the use of bioactive molecules released from MSCs has been described as a novel treatment strategy because of its low tumorigenicity and immunogenicity [5]. Among the trophic factors of MSCs, exosomes have attracted much attention as new mediators in cell-to-cell interactions [6]. Exosomes, one of the extracellular vesicles secreted by cells, are cup-shaped lipid-bilayer nano-sized vesicles (40$120 \mathrm{~nm}$ ). Exosomes originating from the endosomal pathway contain biological factors such as proteins, lipids, and nucleic acids that mediate intercellular communication [7]. MSCderived exosomes can promote phenotypic and functional changes in the surrounding cells and microenvironment by inducing the activation of regenerative events [8]. Recent studies have also demonstrated that exosomes play important roles in the regulation of inflammatory phenotypes via M2 macrophage polarization $[9,10]$. Therefore, an exact understanding of the immunomodulatory effects of exosomes is essential to develop more efficient therapeutic strategies for curing intractable or chronic inflammatory diseases. 
It has been demonstrated that MSC-derived exosomes can upregulate anti-inflammatory factors IL-10 and TGF- $\beta$ and downregulate inflammatory factors IL-6 and TNF $\alpha$ $[11,12]$. In the present study, we examined the antiinflammatory properties of exosomes secreted from adipose MSCs, using fibroblasts and THP-1 cells treated with proinflammatory cytokines interferon gamma (IFN $\gamma$ ) and tumor necrosis factor alpha $(\mathrm{TNF} \alpha)$. We conducted experiments to investigate whether the exosomes could induce anti-inflammatory environments in these models.

\section{Materials and Methods}

2.1. Flow Cytometry. Human adipose tissue-derived MSCs were obtained from healthy donors with the approval from the research ethics committee of Severance Hospital of Yonsei University, Seoul, Republic of Korea, following informed consent (approval no. 4-2019-0060). For surface marker analysis, cultured MSCs were harvested as single cells and incubated with fluorescein isothiocyanate (FITC) or phycoerythrin- (PE-) conjugated antibodies (BD Biosciences Pharmingen, San Diego, CA, USA). The antibodies used in this study were anti-CD14, anti-CD29, anti-CD31, anti-CD34, anti-CD44, anti-CD45, anti-CD73, anti-CD90, anti-CD105, and anti-CD106. Data were acquired using a flow cytometer (Cytomics Flow Cytometer, Beckman Coulter, Fullerton, CA, USA). FITC- and PE-conjugated isotype control antibodies were used as negative controls.

2.2. Isolation of Exosomes. Exosomes were isolated from MSCs using the ExoQuick-TC exosome precipitation reagent, according to the manufacturer's protocols (System Biosciences, CA, USA). Briefly, $5 \mathrm{~mL}$ culture medium was transferred to a $15 \mathrm{~mL}$ conical tube after centrifugation at $1500 \times g$ for $5 \mathrm{~min}$ at RT to remove dead cells and cell debris. Thereafter, the $1 \mathrm{~mL}$ ExoQuick-TC reagent was added to the medium supernatant and mixed via four inversions. The mixture was incubated overnight at $4^{\circ} \mathrm{C}$. The next day, the mixture was centrifuged at $1500 \times g$ for $30 \mathrm{~min}$ to pellet the exosomes. The exosome pellet was suspended in phosphate-buffered saline after removing the supernatant. Following quantification using a BCA protein assay kit (Invitrogen, Carlsbad, CA, USA), exosomes were stored at $-80^{\circ} \mathrm{C}$ until use. Ten micrograms per milliliter of exosomes was used for the experiments. The morphology of isolated exosomes was observed using transmission electron microscopy (TEM) (JEM-1011, JEOL, Japan). Briefly, a formvar-carbon-coated EM grid was placed formvar-side down on top of an exosome drop for $1 \mathrm{~min}$. Thereafter, the grid was placed on a drop of $2 \%$ uranyl acetate and blotted with filter paper. Finally, excess uranyl acetate was removed.

2.3. Western Blotting. The western blot assay was performed as reported previously [9]. Briefly, following boiling at $95^{\circ} \mathrm{C}$ for $5 \mathrm{~min}$, exosomes were separated on $12 \%$ gradient precast gels via sodium dodecyl sulfate polyacrylamide gel electrophoresis and transferred onto a polyvinylidene difluoride membrane (Bio-Rad Laboratories, Redmond, WA, USA). Following blocking with 5\% skim milk in Tris-buffered saline with Tween, the membrane was incubated overnight at $4^{\circ} \mathrm{C}$ with the primary antibodies CD9 (Abcam, Cambridge, MA, USA; $1: 400$ ) and CD63 (Abcam; $1: 500$ ). The next day, the membrane was incubated with HRP-conjugated secondary antibodies and anti-mouse and anti-rabbit IgG (Invitrogen; $1: 1000)$ at RT for $1 \mathrm{~h}$. Following incubation with enhanced chemiluminescence substrate (Bio-Rad) for $1 \mathrm{~min}$, the expression was detected using an LAS4000 mini system (GE Healthcare, Uppsala, Sweden).

2.4. Cell Proliferation Assay. Fibroblasts were seeded at a density of $1 \times 10^{3} /$ well in a 96-well plate (BD Falcon, USA) to analyze cell proliferation. When cells were plated, IFN $\gamma$ and $\mathrm{TNF} \alpha$ were added to each well (10-40 ng/mL). As previously described, the growth activity was determined using a WST8-based EZ-Cytox kit (Daeil Lab, Seoul, Korea) [13]. Two days later, cell proliferation was analyzed according to the manufacturer's instructions. Briefly, the EZ-Cytox reagent was added to each well and incubated for $3 \mathrm{~h}$. Following shaking for $1 \mathrm{~min}$, the absorbance was measured at $450 \mathrm{~nm}$ using a microplate reader (Molecular Devices, San Jose, CA, USA). Untreated cells were used as controls. To optimize the concentration of melatonin, MSCs were treated with varying concentrations $(0.5-50 \mu \mathrm{M})$ of melatonin. Proliferation rates were evaluated using an EZ-Cytox kit after $3 \mathrm{~d}$. Untreated cells ( $0 \mu \mathrm{M}$ of melatonin) were used as controls.

2.5. Real-Time PCR Assay. Quantitative PCR was performed as described previously [9]. Briefly, total RNA was isolated using the RiboEx reagent (GeneAll, Seoul, Korea). Extracted RNA was reverse transcribed into cDNA using Maxime RT PreMix, according to the manufacturer's protocols (iNtRON, Seongnam, Korea). PCR was performed using the LightCycler 480 SYBR Green I Master mix on a LightCycler 480 System under the following conditions: $95^{\circ} \mathrm{C}$ for $5 \mathrm{~min}, 95^{\circ} \mathrm{C}$ for $10 \mathrm{~s}, 45 \mathrm{cycles}$ of $60^{\circ} \mathrm{C}$ for $20 \mathrm{~s}$, and $72^{\circ} \mathrm{C}$ for $15 \mathrm{~s}$. The primer sequences used are listed in Table 1 . Differences between the crossing point $(\mathrm{Cp})$ values of glyceraldehyde 3-phosphate dehydrogenase (GAPDH) and target genes were used to calculate $\triangle \mathrm{Cp}$ values. Relative expression levels were determined using the following formula: relative expression level $=2^{-(\mathrm{S} \Delta \mathrm{Cp}-\mathrm{C} \Delta \mathrm{Cp})}$. For miRNA reactions, PCR was performed at $95^{\circ} \mathrm{C}$ for $10 \mathrm{~min}$, followed by 40 cycles of $95^{\circ} \mathrm{C}$ for $15 \mathrm{~s}, 60^{\circ} \mathrm{C}$ for $1 \mathrm{~min}$, and $72^{\circ} \mathrm{C}$ for $10 \mathrm{~s}$. U6 snRNA was used as an internal control.

2.6. Statistical Analysis. Data are expressed as the mean \pm standard deviation. Statistical analysis was performed using SPSS 18 (SPSS Inc., Chicago, IL, USA). Data were analyzed using one-way analysis of variance followed by Bonferroni's correction or Student's $t$-test. Statistical significance was set at $P<0.05$.

\section{Results}

3.1. Identification of MSCs and Exosomes. MSCs were isolated from the adipose tissue of healthy human donors. Cultured MSCs were examined and photographed under an inverted microscope. The adherent cells exhibited spindle 
TABle 1: Primers used for real-time PCR.

\begin{tabular}{|c|c|}
\hline Name & Sequence \\
\hline GAPDH & $\begin{array}{l}\text { F: ACCCACTCCTCCACCTTTGA } \\
\text { R: CTGTTGCTGTAGCCAAATTCGT }\end{array}$ \\
\hline IL-6 & $\begin{array}{l}\text { F: AGACAGCCACTCACCTCTTCAG } \\
\text { R: TTCTGCCAGTGCCTCTTTGCTG }\end{array}$ \\
\hline IL-8 & $\begin{array}{l}\text { F: AAGAGAGCTCTGTCTGGACC } \\
\text { R: GATATTCTCTTGGCCCTTGG }\end{array}$ \\
\hline TSG-6 & $\begin{array}{l}\text { F: GGTGTGTACCACAGAGAAGCA } \\
\text { R: GGGTTGTAGCAATAGGCATCC }\end{array}$ \\
\hline TGF-ß1 & $\begin{array}{l}\text { F: TACCTGAACCCGTGTTGCTCTC } \\
\text { R: GTTGCTGAGGTATCGCCAGGAA }\end{array}$ \\
\hline CD163 & $\begin{array}{l}\text { F: CGGCTGCCTCCACCTCTAAGT } \\
\text { R: ATGAAGATGCTGGCGTGACA }\end{array}$ \\
\hline $\mathrm{TNF} \alpha$ & $\begin{array}{l}\text { F: TTGAGGGTTTGCTACAACATGGG } \\
\text { R: GCTGCACTTTGGAGTGATCG }\end{array}$ \\
\hline Arg-1 & $\begin{array}{l}\text { F: ACAGTTTGGCAATTGGAAGCA } \\
\text { R: CACCCAGATGACTCCAAGATCAG }\end{array}$ \\
\hline CD206 & $\begin{array}{l}\text { F: TTCGGACACCCATCGGAATTT } \\
\text { R: CACAAGCGCTGCGTGGAT }\end{array}$ \\
\hline IL-10 & $\begin{array}{l}\text { F: TCTCCGAGATGCCTTCAGCAGA } \\
\text { R: TCAGACAAGGCTTGGCAACCCA }\end{array}$ \\
\hline U6 & $\begin{array}{l}\text { F: CTCGCTTCGGCAGCACATATACT } \\
\text { R: ACGCTTCACGAATTTGCGTGTC }\end{array}$ \\
\hline miR-34a & $\begin{array}{c}\text { F: ACACTCCAGCTGTGACTGGTTGACCAGA } \\
\text { R: CTCAACTGGTGTCGTGGA }\end{array}$ \\
\hline miR-124 & $\begin{array}{l}\text { F: TAAGGCACGCGGTGAATGCC } \\
\text { R: GATTGAATCGAGCACCAGTTAC }\end{array}$ \\
\hline miR-135b & $\begin{array}{l}\text { F: GCTTATGGCTTTTCATTCCT } \\
\text { R: GTGCAGGGTCCGAGGT }\end{array}$ \\
\hline
\end{tabular}

fibroblast-like morphology (Figure 1(a)). Flow cytometry results showed that the cells were positive for MSC markers CD29, CD44, CD73, CD90, and CD105, but not for hematopoietic/endothelial markers CD14, CD31, CD34, CD45, and CD106 (Figure 1(b)). These results demonstrated that the cultured cells showed typical MSC characteristics.

Subsequently, exosomes isolated from MSCs were characterized using TEM and western blotting. Morphological characterization showed the existence of small membrane vesicles measuring 57.77-62.02 nm (Figure 1(c)). Western blotting revealed that the isolated vesicles were positive for the expression of exosomal markers such as CD9 and CD63 (Figure 1(d)). TEM and western blotting results revealed that the vesicles isolated from MSCs were exosomes.

3.2. Effects of Exosomes on IFN $\gamma$ and TNF $\alpha$-Treated Fibroblasts. To determine whether pro-inflammatory cytokines affect the morphology and proliferation activity of fibroblasts, cells were cultured with IFN $\gamma$ and TNF $\alpha$ at concentrations from 10 to $40 \mathrm{ng} / \mathrm{mL}$ for $48 \mathrm{~h}$. We observed that treatment with pro-inflammatory cytokines induced an enlarged senescent-like morphology at high concentrations ( $40 \mathrm{ng} / \mathrm{mL} \mathrm{IFN} \gamma$ and $40 \mathrm{ng} / \mathrm{mL} \mathrm{TNF} \alpha$ ) (Figure 2(a)). Moreover, the concentration of $40 \mathrm{ng} / \mathrm{mL}$ cytokines resulted in the lowest growth rate of fibroblasts (Figure 2(b)). Therefore, this concentration was selected to induce inflammation.
To evaluate the effects of exosomes on pro-inflammatory cytokine-treated fibroblasts, the inflammation-related gene expression was analyzed using real-time PCR. Our data show that the expression levels of pro-inflammatory cytokines $\mathrm{TNF} \alpha$ and IL- 6 genes increased in the presence of cytokines and decreased upon exosome $(10 \mu \mathrm{g} / \mathrm{mL})$ treatment (Figure 2(c)). Alternatively, the gene expression level of the anti-inflammatory factor TSG-6 did not change despite exosome treatment (Figure 2(c)). These results indicate that exosomes exert anti-inflammatory effects by downregulating pro-inflammatory genes.

3.3. Effects of Exosomes on Proinflammatory CytokineTreated THP-1 Cells. THP-1 cells are a human monocytic cell line derived from an acute monocytic leukemia patient. THP-1 cells respond to diverse inflammatory cytokines and are used as an inflammation model in vitro [14]. To assess the effects of exosomes on inflammation in greater detail, we used the THP-1 cell line. Stimulation with IFN $\gamma$ and $\mathrm{TNF} \alpha$ increased the expression of TNF $\alpha$, IL-6, and IL-8, which are associated with pro-inflammatory M1 macrophages; however, the expression of TGF- $\beta 1$ and TSG-6, which are related to anti-inflammatory M2 macrophages, was significantly enhanced only in the presence of exosomes (Figure 3). Collectively, these results indicate that exosomes can inhibit inflammatory responses induced by $\operatorname{IFN} \gamma$ and $\mathrm{TNF} \alpha$.

3.4. Effects of Melatonin on MSC Proliferation and Characterization of Melatonin-Stimulated Exosomes (MelExosome). Different concentrations $(0-50 \mu \mathrm{M})$ of melatonin were applied to cells to investigate its effects on THE MSC proliferation activity and its optimal concentration. Overall, the growth rates of cells increased after melatonin treatment regardless of concentration (Figure 4(a)). At varying concentrations, $10 \mu \mathrm{M}$ of melatonin resulted in the highest growth rate after $3 \mathrm{~d}$ of culture (Figure 4(a)). These results are in line with those of a previous study [15]. Therefore, melatonin at $10 \mu \mathrm{M}$ was used as the optimal concentration.

To identify changes depending on melatonin treatment, the exosome morphology derived from Mel-Exosome and untreated cells was confirmed by TEM (Figure 4(b)). The size of isolated exosomes was in the range of $30-50 \mathrm{~nm}$, and the CD9 exosomal marker was detected in both Mel-Exosome and untreated exosomes, indicating that exosomes were not influenced by melatonin treatment (Figure 4(b)).

3.5. Effects of Melatonin-Stimulated Exosomes on Pronflammatory Cytokine-Treated THP-1 Cells. Following treatment with $40 \mathrm{ng} / \mathrm{mL}$ IFN $\gamma$ and TNF $\alpha$, THP- 1 cells were equivalently treated with $10 \mu \mathrm{M}, 10 \mu \mathrm{g} / \mathrm{mL}$ exosomes, and $10 \mu \mathrm{g} / \mathrm{mL}$ melatonin $(10 \mu \mathrm{M})$-Mel-Exosome to evaluate the ability of melatonin-stimulated exosomes. First, we evaluated macrophage differentiation from THP-1 cells by real-time PCR. We detected elevated gene expression levels of M2 macrophage markers CD206 and CD163 under all conditions (Figure 5). Notably, we observed that their expression levels were significantly upregulated in melatonin-treated exosomes (Figure 5). However, only melatonin-treated exosomes (Mel- 


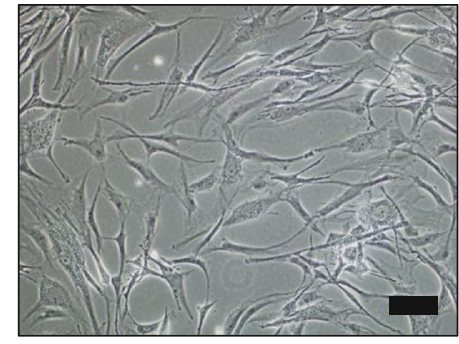

(a)
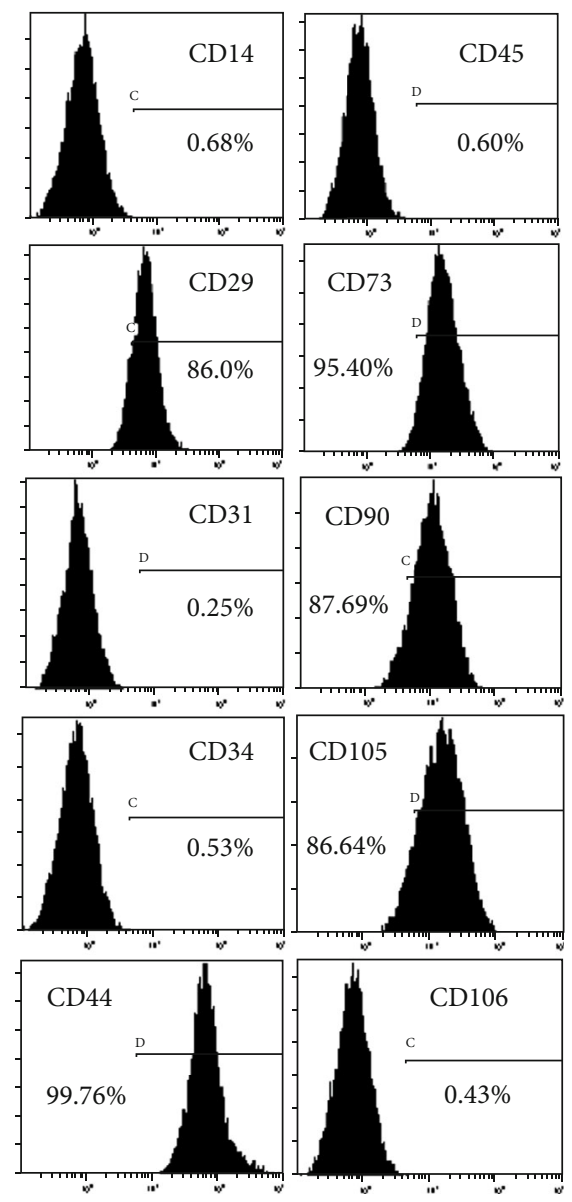

(b)
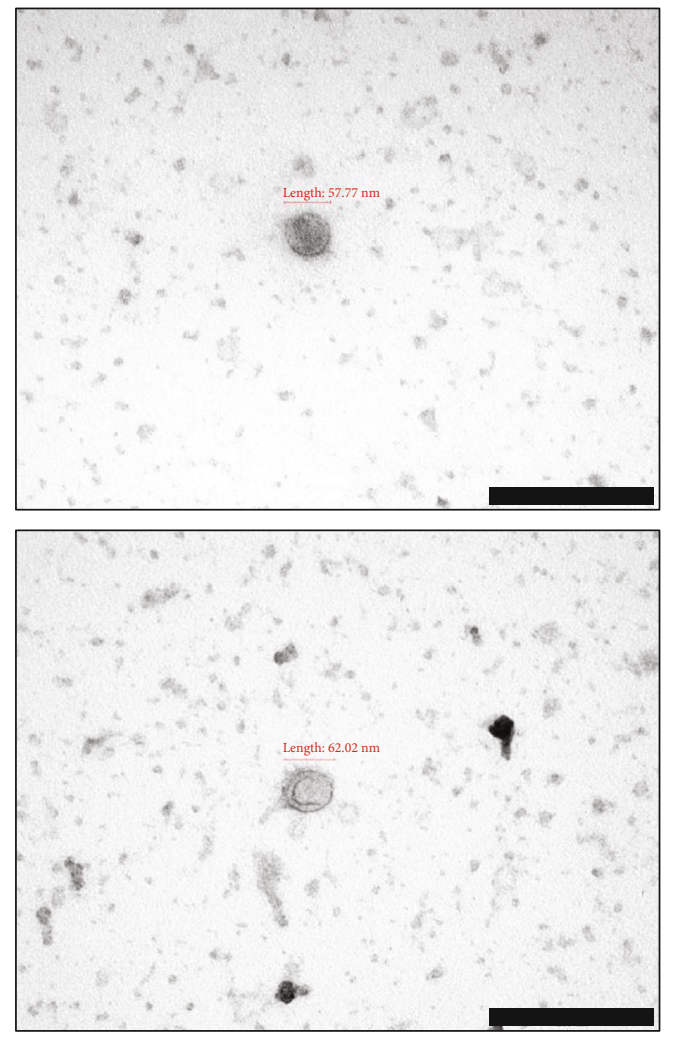

(c)

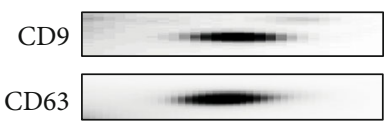

(d)

FIGURE 1: Isolation and characterization of adipose MSCs and adipose MSC-derived exosomes. (a) Spindle-shaped fibroblast-like morphology observed via an inverted microscope ( $\times 100$ magnification). (b) Surface markers of cultured cells showing the typical pattern of MSCs. (c) TEM image of isolated exosomes (scale bar $=200 \mathrm{~nm}$ ). (d) The expression of CD9 and CD63 in exosomes was detected using western blotting. A representative independent experiment is shown. MSCs: mesenchymal stem cells; TEM: transmission electron microscopy.

Exosome) were able to upregulate the expression of the M2 macrophage marker Arg-1 (Figure 5).

Second, we detected the expression levels of antiinflammatory genes TGF- $\beta 1$, TSG- 6 , and IL- 10 . We noticed that the TSG-6 gene expression in the exosomes and MelExosome groups significantly increased, and that of TGF$\beta 1$ was only markedly expressed in melatonin-treated exosomes (Figure 5). Notably, the IL-10 gene expression was significant only after melatonin and Mel-Exosome treatment, indicating that the IL-10 expression was affected by melatonin (Figure 5). Collectively, our data showed that exosomes could induce M2 polarization in agreement with antiinflammatory changes, and Mel-Exosome could strongly promote these effects, implying their applicability in inflammation-related diseases.
Finally, we analyzed specific miRNAs miR-34a, miR-124, and miR-135b of anti-inflammatory M2 macrophages between exosomes and Mel-Exosome. As shown in Figure 6, the expression of miR-34a, miR-124, and miR-135b significantly increased in melatonin-stimulated exosomes compared with untreated exosomes. The results of these studies verify that melatonin-stimulated exosomes (Mel-Exosome) have powerful effects on M2 polarization-mediated antiinflammation.

\section{Discussion}

The therapeutic effects of MSCs are associated with the transfer of their paracrine factors, which regulate cellular functionality [16]. Previously, Jeong et al. showed that 

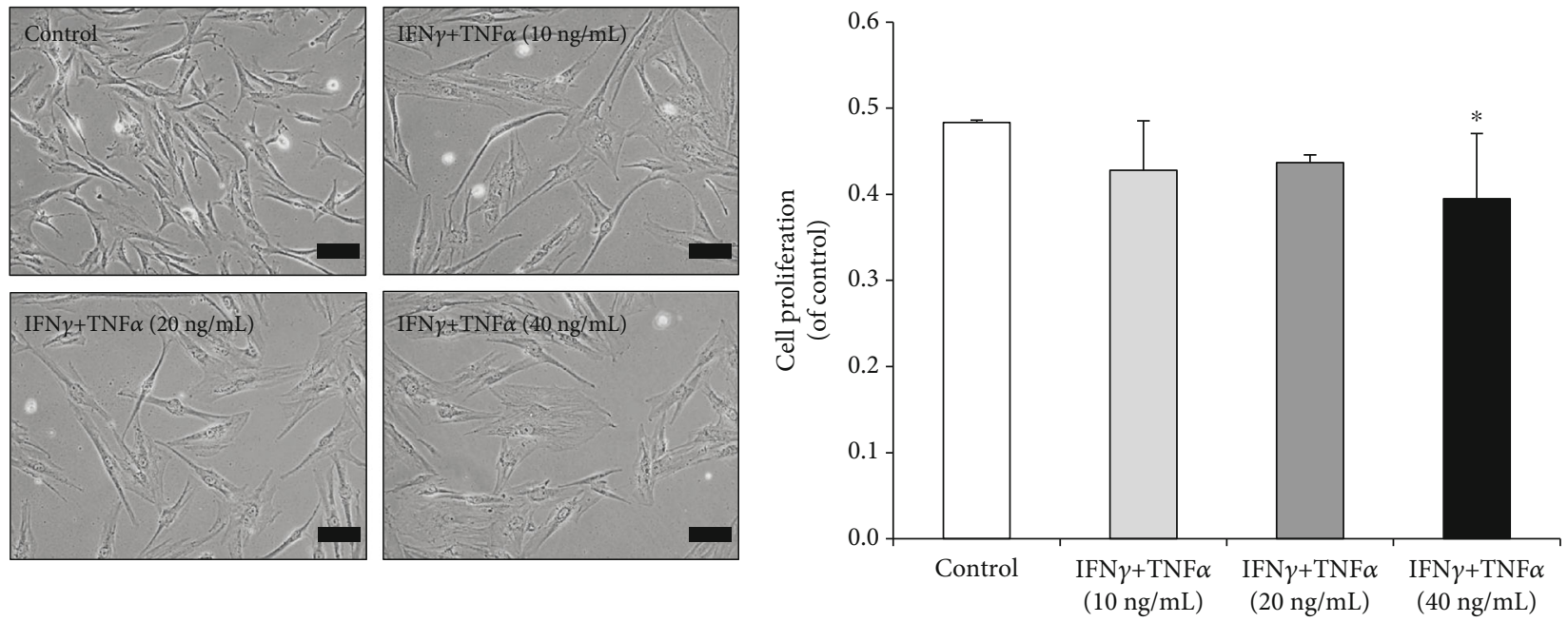

(a)

(b)
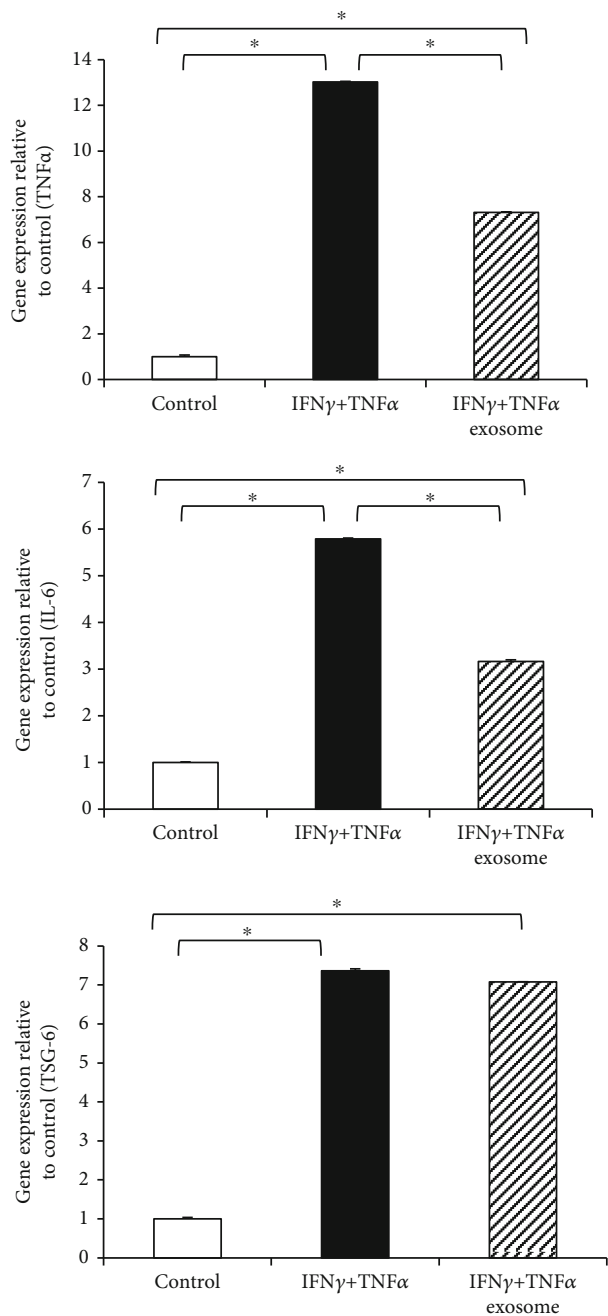

(c)

FIgURE 2: Treatment with combination of IFN $\gamma$ and TNF $\alpha$ to induce inflammation in fibroblasts. (a) Optical images of MSCs stimulated with IFN $\gamma$ and TNF $\alpha$ at concentrations of 10,20, and $40 \mathrm{ng} / \mathrm{mL}$ for $48 \mathrm{~h}$. A representative image based on three independent experiments is shown ( $\times 200$ magnification). (b) Cell proliferation evaluated using the WST-1-based colorimetric assay. ${ }^{*} P<0.05$ compared with the control group. (c) mRNA expression levels of inflammation-associated genes TNF $\alpha$, IL6, and TSG6, analyzed using real-time PCR. Data are represented as the mean \pm SD of three independent experiments. IFN $\gamma$ : interferon gamma; TNF $\alpha$ : tumor necrosis factor alpha; MSCs: mesenchymal stem cells. 

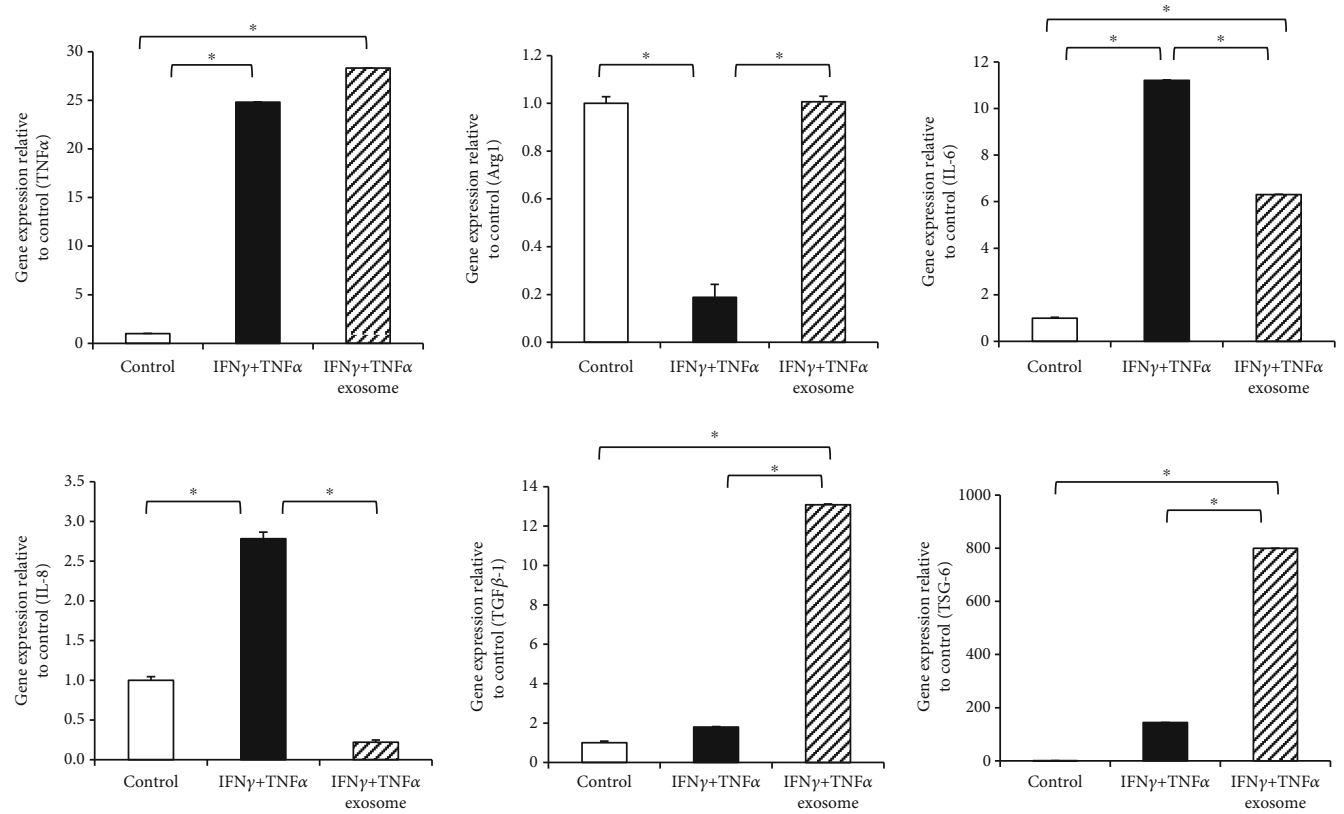

FIGURE 3: Effects of exosomes on pro-inflammatory cytokine-treated THP-1 cells. mRNA expression levels after pro-inflammatory cytokine (IFN $\gamma$ and TNF $\alpha$ ) treatment were determined using real-time PCR and normalized with GAPDH mRNA levels. Untreated THP-1 cells were used as the control. Data are represented as the mean \pm SD of three independent experiments ${ }^{*} P<0.05$. IFN $\gamma$ : interferon gamma; TNF $\alpha$ : tumor necrosis factor alpha; GAPDH: glyceraldehyde 3-phosphate dehydrogenase.

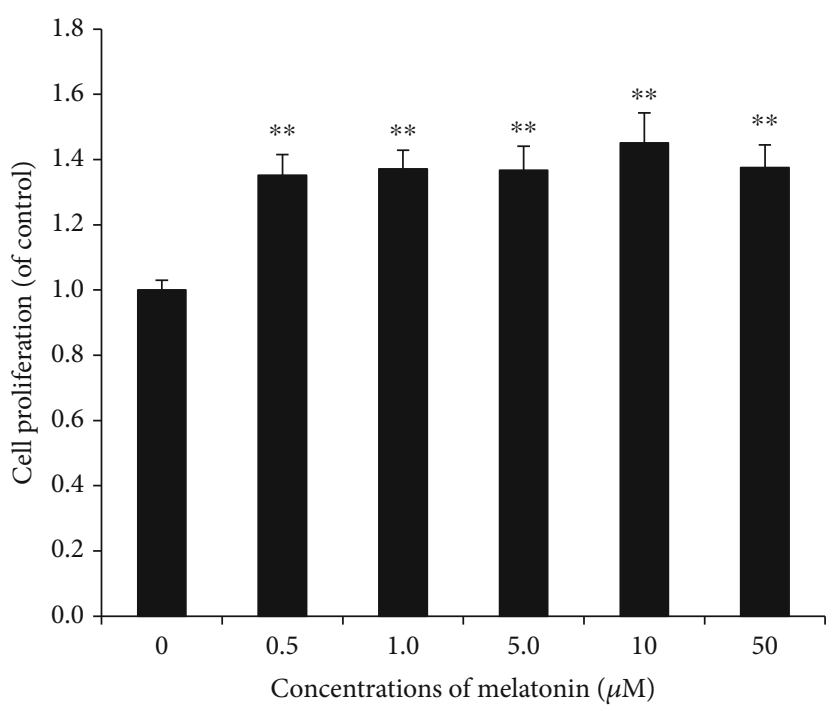

(a)
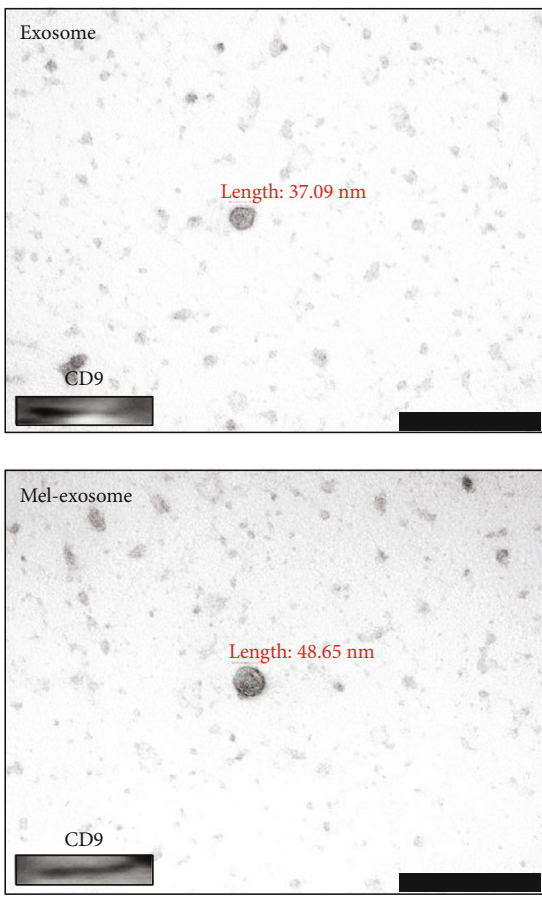

(b)

FIgURE 4: Effects of melatonin on the MSC growth activity and exosome characterization. (a) MSCs were cultured with different concentrations $(0-50 \mu \mathrm{M})$ of melatonin for $72 \mathrm{~h}$. The proliferation rates of cells were evaluated using the proliferation assay kit (EZ-cytox assay). Data are represented as the mean $\pm S D$ of three independent experiments ${ }^{* *} P<0.01$. (b) The morphology of untreated exosomes and melatonin-stimulated exosomes (Mel-Exosome) was confirmed by TEM (scale bar $=200 \mathrm{~nm}$ ). The exosomal marker CD9 was detected in untreated exosomes and melatonin-stimulated exosomes (Mel-Exosome) by western blotting. A representative independent experiment is shown. MSC: mesenchymal stem cell; TEM: transmission electron microscopy. 

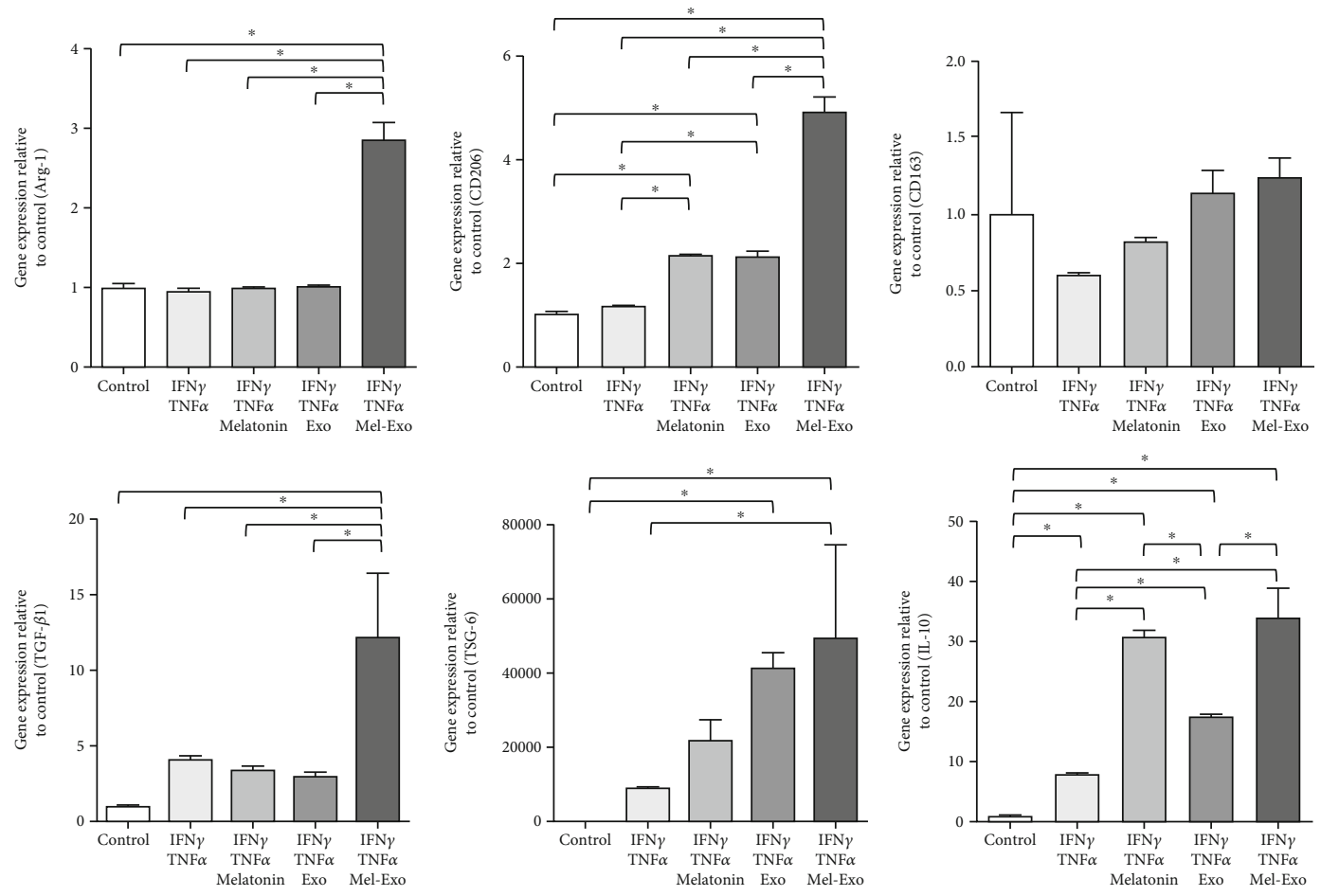

FIGURE 5: Effects of untreated exosomes and melatonin-stimulated exosomes (Mel-Exosome) on pro-inflammatory cytokines-treated THP-1 cells. Relative mRNA expression levels after pro-inflammatory cytokine (IFN $\gamma$ and TNF $\alpha$ ) treatment were determined using real-time PCR and normalized with GAPDH mRNA levels. Untreated THP-1 cells were used as control. Data are represented as the mean \pm SD of three independent experiments. ${ }^{*} P<0.05$. IFN $\gamma$ : interferon gamma; TNF $\alpha$ : tumor necrosis factor alpha; GAPDH: glyceraldehyde 3-phosphate dehydrogenase.

transplantation of cultured MSCs can form tumors with chromosomal abnormalities in vivo [17]. An important advantage of therapy using exosomes is freedom from clinical risks of transplanted cells due to lower immunogenicity and no risk of tumorigenicity [5]. Recent evidence has shown that adipose-derived MSC exosomes induce M2 macrophages with an anti-inflammatory phenotype [9]. Furthermore, several studies also indicate that MSC-derived exosomes exert anti-inflammatory effects by enhancing anti-inflammatory factors such as IL-10 and TGF- $\beta 1[18,19]$. Based on previous reports, we investigated the potential effects of exosomes derived from adipose MSCs in IFN $\gamma$ and TNF- $\alpha$-treated inflammatory models. Notably, exosomes markedly attenuated inflammation-related genes, including TNF $\alpha$ and IL-6, in pro-inflammatory cytokine-induced fibroblasts. In a proinflammatory cytokine-stimulated THP-1 cellular model, exosome treatment resulted in the notable induction of an M2 macrophage-mediated anti-inflammatory environment. The changes in the expression of inflammation-related markers coincided with an increase in the expression of Arg1 and TSG- 6 of anti-inflammatory M2 macrophages, suggesting that exosomes could be used as therapeutic agents to control inflammation in the biomedical field.

Melatonin has been reported to play crucial roles in stem cell research [20]. For example, melatonin has been demonstrated to promote stem cell proliferation and therapeutic efficacy by regulating inflammatory conditions [21-23]. However, the synergistic effect of combined melatonin and exosome has been not extensively studied. MSCs stimulated by pro-inflammatory cytokines secrete soluble molecules known as paracrine factors, which promote M2 macrophage polarization expressing Arg-1, CD206, and CD163. Polarized M2 macrophages facilitate anti-inflammatory responses by enhancing TGF- $\beta 1$, TSG-6, and IL-10 [24]. To investigate the effects of melatonin-stimulated exosomes on inflammation, we evaluated the expression of specific genes associated with anti-inflammatory M2 macrophages. The relative gene expression demonstrated that in comparison with untreated exosomes, M2 macrophage-mediated anti-inflammatory changes were reinforced after melatonin-stimulated exosome application. Alternatively, some results showed that exosomes failed to induce substantial anti-inflammatory M2 macrophages. These results might be attributable to incomplete macrophage polarization, consistent with the conclusions of previous findings [9]. It has been reported that melatonin enhances the IL-10 expression to suppress inflammation [25]. Our data demonstrated that the IL-10 expression significantly increased only by melatonin treatment. Therefore, we believe that melatonin-stimulated exosomes have a synergistic effect on the inflammatory environment.

An increasing number of reports indicate that exosomedelivered miRNAs regulate inflammatory responses by targeting mRNAs [26]. To determine whether exosomes exert M2 macrophage-mediated anti-inflammatory effects via specific miRNAs, we analyzed the expression of miRNAs in melatonin-stimulated miRNAs and compared them with 

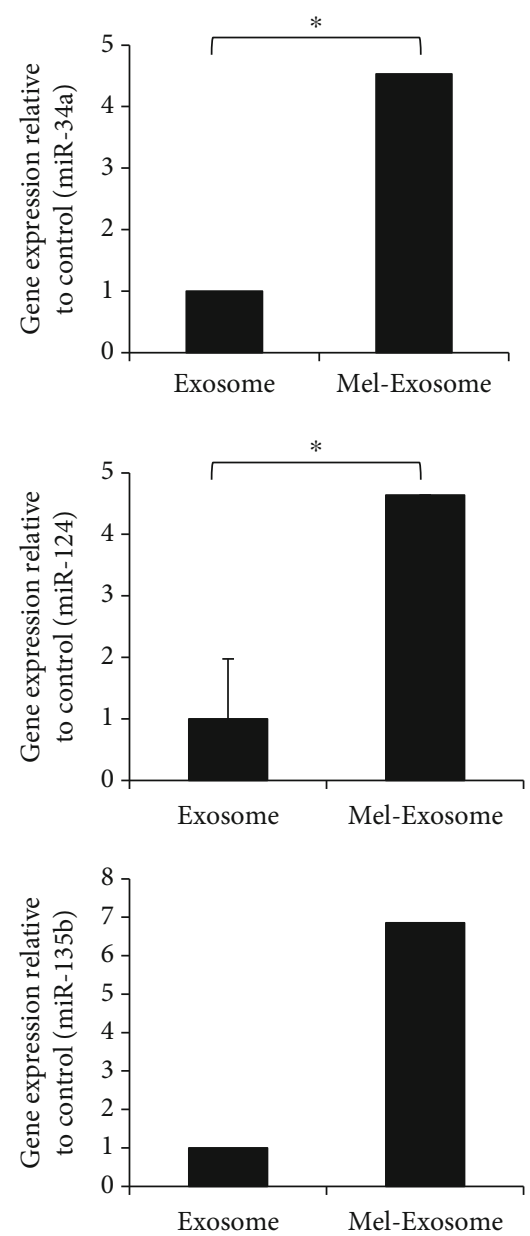

FIgURe 6: Expression of miR-34a, miR-124, and miR-135b in exosomes. The relative expression levels of miRNAs were analyzed in melatonin-stimulated exosomes (Mel-Exosome) compared with untreated exosomes by real-time PCR. Data are represented as the mean \pm SD of three independent experiments. ${ }^{*} P<0.05$.

those in untreated exosomes. MiR-34a has been reported to promote M2 macrophages and miR-124 reduced M1 polarization and enhanced M2 polarization [27, 28]. In addition, the anti-inflammatory properties of MSCs have been linked to miR-135b-mediated M2 polarization [29]. Thus, we confirmed that miR-34a, miR-124, and miR-135b were significantly upregulated in melatonin-stimulated exosomes. The upregulation of these miRNAs strongly supports a significant increase in Arg-1, CD206, CD163, TGF- $\beta 1$, TSG-6, and IL10 gene expression, which contributed to the promotion of an anti-inflammatory microenvironment. Hence, our data suggest that melatonin-stimulated exosomes may be a promising therapeutic tool to suppress inflammation caused by chronic inflammatory diseases or autoimmune disorders, even if the mechanisms are not fully understood.

\section{Conclusions}

Inflammation is a basic pathological mechanism of complex biological responses in a variety of diseases. The inflammatory reaction involves complex interactions of macrophages, neutrophils, and lymphocytes, which are called inflammatory cells [30]. Although exosomes play a crucial role in modulating inflammatory responses, the mechanisms and signaling pathways related to the activation or inactivation of diverse inflammatory mediators and cytokines remain unclear. Moreover, additional confirmatory data need to be acquired using miRNA sequencing and analysis at the protein level. Additionally, further investigations are necessary to explore the synergistic effect of combined melatonin and exosomes using animal models. In conclusion, the findings of the present study contribute to the development of stem cell-based cell-free therapies by providing valuable information that can be used for inflammation-related diseases.

\section{Data Availability}

The data used to support the findings of this study are available from the corresponding author upon request.

\section{Conflicts of Interest}

The authors declare that they have no known competing financial interests or personal relationships that could have appeared to influence the work reported in this paper.

\section{Acknowledgments}

This work was supported by the National Research Foundation of Korea (NRF) grant funded by the Korean government (MSIT) (June Seok Heo, No. 2019R1C1C1007036 and Jinkwan Kim, No. 2018R1D1A1B07050071).

\section{References}

[1] J. S. Heo, Y. Choi, H. S. Kim, and H. O. Kim, "Comparison of molecular profiles of human mesenchymal stem cells derived from bone marrow, umbilical cord blood, placenta and adipose tissue," International Journal of Molecular Medicine, vol. 37, no. 1, pp. 115-125, 2016.

[2] S. S. Iyer and M. Rojas, "Anti-inflammatory effects of mesenchymal stem cells: novel concept for future therapies," Expert Opinion on Biological Therapy, vol. 8, no. 5, pp. 569-581, 2008.

[3] L. T. Wang, C. H. Ting, M. L. Yen et al., "Human mesenchymal stem cells (MSCs) for treatment towards immune- and inflammation-mediated diseases: review of current clinical trials," Journal of Biomedical Science, vol. 23, no. 1, p. 76, 2016.

[4] M. Z. Ratajczak, M. Kucia, T. Jadczyk et al., "Pivotal role of paracrine effects in stem cell therapies in regenerative medicine: can we translate stem cell-secreted paracrine factors and microvesicles into better therapeutic strategies?," Leukemia, vol. 26, no. 6, pp. 1166-1173, 2012.

[5] K. Yin, S. Wang, and R. C. Zhao, "Exosomes from mesenchymal stem/stromal cells: a new therapeutic paradigm," Biomarker Research, vol. 7, no. 1, 2019.

[6] G. K. Patel, M. A. Khan, H. Zubair et al., "Comparative analysis of exosome isolation methods using culture supernatant for optimum yield, purity and downstream applications," Scientific Reports, vol. 9, no. 1, p. 5335, 2019.

[7] F. Vakhshiteh, F. Atyabi, and S. N. Ostad, "Mesenchymal stem cell exosomes: a two-edged sword in cancer therapy," International Journal of Nanomedicine, vol. 14, pp. 2847-2859, 2019. 
[8] G. Camussi, M. C. Deregibus, and C. Tetta, "Paracrine/endocrine mechanism of stem cells on kidney repair: role of microvesicle-mediated transfer of genetic information," Current Opinion in Nephrology and Hypertension, vol. 19, no. 1, pp. 7-12, 2010.

[9] J. S. Heo, Y. Choi, and H. O. Kim, “Adipose-derived mesenchymal stem cells promote M2 macrophage phenotype through exosomes," Stem Cells International, vol. 2019, Article ID 7921760, 10 pages, 2019.

[10] D. K. Singla, T. A. Johnson, and Z. Tavakoli Dargani, "Exosome treatment enhances anti-inflammatory M2 macrophages and reduces inflammation-induced pyroptosis in doxorubicin-induced cardiomyopathy," Cells, vol. 8, no. 10, p. 1224, 2019.

[11] R. Blazquez, F. M. Sanchez-Margallo, O. de la Rosa et al., "Immunomodulatory potential of human adipose mesenchymal stem cells derived exosomes on in vitro stimulated T cells," Frontiers in Immunology, vol. 5, p. 556, 2014.

[12] B. Zhang, Y. Yin, R. C. Lai, S. S. Tan, A. B. H. Choo, and S. K. Lim, "Mesenchymal stem cells secrete immunologically active exosomes," Stem Cells and Development, vol. 23, no. 11, pp. 1233-1244, 2014.

[13] J. S. Heo, S. G. Lee, and H. O. Kim, "The flavonoid glabridin induces OCT4 to enhance osteogenetic potential in mesenchymal stem cells," Stem Cells International, vol. 2017, Article ID 6921703, 10 pages, 2017.

[14] L. Yang, H. Guo, Y. Li et al., "Oleoylethanolamide exerts anti-inflammatory effects on LPS-induced THP-1 cells by enhancing PPAR $\alpha$ signaling and inhibiting the NF- $\kappa \mathrm{B}$ and ERK1/2/AP-1/STAT3 pathways," Scientific Reports, vol. 6, no. 1 , article 34611, 2016.

[15] J. S. Heo, S. Pyo, J. Y. Lim et al., "Biological effects of melatonin on human adipose-derived mesenchymal stem cells," International Journal of Molecular Medicine, vol. 44, no. 6, pp. 22342244, 2019.

[16] W. S. Park, S. Y. Ahn, S. I. Sung, J. Y. Ahn, and Y. S. Chang, "Strategies to enhance paracrine potency of transplanted mesenchymal stem cells in intractable neonatal disorders," Pediatric Research, vol. 83, no. 1-2, pp. 214-222, 2018.

[17] J. O. Jeong, J. W. Han, J. M. Kim et al., "Malignant tumor formation after transplantation of short-term cultured bone marrow mesenchymal stem cells in experimental myocardial infarction and diabetic neuropathy," Circulation Research, vol. 108, no. 11, pp. 1340-1347, 2011.

[18] R. Dong, Y. Liu, Y. Yang, H. Wang, Y. Xu, and Z. Zhang, "MSC-derived exosomes-based therapy for peripheral nerve injury: a novel therapeutic strategy," BioMed Research International, vol. 2019, Article ID 6458237, 12 pages, 2019.

[19] P. Xian, Y. Hei, R. Wang et al., "Mesenchymal stem cellderived exosomes as a nanotherapeutic agent for amelioration of inflammation-induced astrocyte alterations in mice," Theranostics, vol. 9, no. 20, pp. 5956-5975, 2019.

[20] Y. Shuai, L. Liao, X. Su et al., "Melatonin treatment improves mesenchymal stem cells therapy by preserving stemness during long-term in vitro expansion," Theranostics, vol. 6, no. 11, pp. 1899-1917, 2016.

[21] C. Hu and L. Li, "Melatonin plays critical role in mesenchymal stem cell-based regenerative medicine in vitro and in vivo," Stem Cell Research \& Therapy, vol. 10, no. 1, p. 13, 2019.

[22] H. Kato, G. Tanaka, S. Masuda et al., "Melatonin promotes adipogenesis and mitochondrial biogenesis in 3T3-L1 preadi- pocytes," Journal of Pineal Research, vol. 59, no. 2, pp. 267275, 2015.

[23] Y. Tang, B. Cai, F. Yuan et al., "Melatonin pretreatment improves the survival and function of transplanted mesenchymal stem cells after focal cerebral ischemia," Cell Transplantation, vol. 23, no. 10, pp. 1279-1291, 2014.

[24] G. Zheng, M. Ge, G. Qiu, Q. Shu, and J. Xu, "Mesenchymal stromal cells affect disease outcomes via macrophage polarization," Stem Cells International, vol. 2015, Article ID 989473, 11 pages, 2015.

[25] S. J. Chen, S. H. Huang, J. W. Chen et al., "Melatonin enhances interleukin-10 expression and suppresses chemotaxis to inhibit inflammation in situ and reduce the severity of experimental autoimmune encephalomyelitis," International Immunopharmacology, vol. 31, pp. 169-177, 2016.

[26] M. Alexander, R. Hu, M. C. Runtsch et al., "Exosome-delivered microRNAs modulate the inflammatory response to endotoxin," Nature Communications, vol. 6, no. 1, 2015.

[27] G. Liu and E. Abraham, "MicroRNAs in immune response and macrophage polarization," Arteriosclerosis, Thrombosis, and Vascular Biology, vol. 33, no. 2, pp. 170-177, 2013.

[28] J. Zhou, Z. Li, T. Wu, Q. Zhao, Q. Zhao, and Y. Cao, "LncGBP9/miR-34a axis drives macrophages toward a phenotype conducive for spinal cord injury repair via STAT1/STAT6 and SOCS3," Journal of Neuroinflammation, vol. 17, no. 1, p. 134, 2020.

[29] R. Domenis, A. Cifù, S. Quaglia et al., "Pro inflammatory stimuli enhance the immunosuppressive functions of adipose mesenchymal stem cells-derived exosomes," Scientific Reports, vol. 8, no. 1, article 13325, 2018.

[30] A. Tedgui and Z. Mallat, "Anti-inflammatory mechanisms in the vascular wall," Circulation Research, vol. 88, no. 9, pp. 877-887, 2001. 